\title{
INDEPENDENT SETS OF GENERATORS OF PRIME POWER ORDER
}

\author{
ANDREA LUCCHINI AND PABLO SPIGA
}

\begin{abstract}
A subset $X$ of a finite group $G$ is said to be prime-power-independent if each element in $X$ has prime power order and there is no proper subset $Y$ of $X$ with $\langle Y, \Phi(G)\rangle=\langle X, \Phi(G)\rangle$, where $\Phi(G)$ is the Frattini subgroup of $G$. A group $G$ is $\mathcal{B}_{p p}$ if all prime-power-independent generating sets for $G$ have the same cardinality. We prove that, if $G$ is $\mathcal{B}_{p p}$, then $G$ is solvable. Pivoting on some recent results of Krempa and Stocka [10 [16, this yields a complete classification of $\mathcal{B}_{p p}$-groups.
\end{abstract}

\section{INTRODUCTION}

Throughout this paper, all groups are finite. We start this introductory section with some definitions fundamental for our work. Given a group $G$, an element $g \in G$ is said to be a $p p$-element if $g$ has prime power order. A subset $X$ of $G$ is said to be

independent: if $\langle X, \Phi(G)\rangle \neq\langle Y, \Phi(G)\rangle$ for every proper subset $Y$ of $X$ (where as customary we denote by $\Phi(G)$ the Frattini subgroup of $G$ );

pp-independent: if $X$ is independent and each element in $X$ is a $p p$-element; and

$p p$-base: if $X$ is a $p p$-independent generating set for $G$.

Finally, $G$ is said to be a $\mathcal{B}_{p p^{-}}$group if every two $p p$-bases of $G$ have the same cardinality.

The main result of this paper is the following.

Theorem 1.1. If $G$ is a $\mathcal{B}_{p p}$-group, then $G$ is solvable.

Theorem 1.1 gives a solution to Question 1 in 10 in a strong sense. In fact, it yields a complete classification of the $\mathcal{B}_{p p}$-groups. Indeed, Krempa and Stocka [10, 16] have obtained an entirely satisfactory classification of solvable $\mathcal{B}_{p p}$-groups and hence Theorem 1.1 together with the work in [10, 16] gives a classification of all $\mathcal{B}_{p p}$-groups. This classification is easier to formulate for Frattini-free groups, that is, for groups $G$ with $\Phi(G)=1$. (Observe that $G$ is a $\mathcal{B}_{p p}$-group if and only if so is $G / \Phi(G)$.)

Corollary 1.2. Let $G$ be a group with $\Phi(G)=1$. Then $G$ is a $\mathcal{B}_{p p}$-group and if only if one of the following holds:

(1) $G$ is an elementary abelian p-group,

(2) $G=P \rtimes Q$, where $P$ is an elementary abelian p-group, $Q$ is a non-identity cyclic q-group for distinct prime numbers $p$ and $q$ such that $Q$ acts faithfully on $P$ and the $(\mathbb{Z} / p \mathbb{Z})[Q]$-module $P$ is a direct sum of pair-wise isomorphic simple modules,

(3) $G$ is a direct product of groups given in (1) or in (2) with pair-wise coprime orders.

The groups as in (2) are simply refereed to as scalar extensions in [16. We refer the reader to the work of Krempa and Stocka [10, 16] for various motivations on investigating $\mathcal{B}_{p p}$-groups. Broadly speaking, this motivation is rooted on independent generating sets and on generalizations of the Burnside basis theorem; in turn, these motivations are useful for studying groups satisfying the exchange property for bases which is useful for constructing matroids starting from finite groups.

As a bi-product of the arguments used in the proof of Theorem 1.1, we obtain the following result of independent interest. (See Section 2.1 for undefined terminology.)

Theorem 1.3. Let $G$ be a group and denote by $m(G)$ the largest cardinality of an independent generating set of $G$. Then $m(G) \geq a+b$, where $a$ and $b$ are, respectively, the number of non-Frattini and non-abelian factors in a chief series of $G$.

We have verified with a computer computation [1] that the bound in Theorem 1.3 is sharp when $G$ is the automorphism group of the alternating group of degree 6 : here, $m(G)=4, a=3$ and $b=1$. Theorem 1.3 gives a strengthening of the bound $m(G) \geq a$, which was proved in [13. Here, it was also proved that $m(G)=a$ for every solvable group.

The structure of the paper is straightforward. In Section 2 after establishing some notation, and after a short detour through fixed point ratios and spreads, we give some basic results. In Section 3 after establishing a few rather technical results, we prove Theorem 1.1 and Corollary 1.2 . Finally, we prove Theorem 1.3 in Section 4 .

2010 Mathematics Subject Classification. primary 20D10, secondary 20D60, 20 F05.

Key words and phrases. independent sets, generating set, Burnside basis theorem. 


\section{Preliminaries}

2.1. Notation. Given a group $G$, we let $m(G)$ and $m_{p p}(G)$ denote the largest cardinality of an independent generating set of $G$ and of a $p p$-independent generating set for $G$. Since every $p p$-independent generating set is also an independent generating set, we have $m(G) \geq m_{p p}(G)$. In fact, in Lemma 2.3 we show that $m(G)=m_{p p}(G)$.

Let

$$
1=G_{t} \unlhd \cdots \unlhd G_{0}=G
$$

be a chief series for $G$. A factor $G_{i} / G_{i+1}$ is said to be a non-abelian chief factor of $G$ if $G_{i} / G_{i+1}$ is a non-abelian group; moreover, $G_{i} / G_{i+1}$ is said to be a Frattini chief factor of $G$ if $G_{i} / G_{i+1} \leq \Phi\left(G / G_{i+1}\right)$.

The socle of $G$, denoted by soc $G$, is the subgroup generated by the minimal normal subgroups of $G$. In particular, if $\operatorname{soc} G$ is a minimal normal subgroup of $G$ (that is, $G$ has a unique minimal normal subgroup), then $G$ is said to be monolithic.

Let $G$ be a monolithic group with socle $N$. Following the notation in [14, we define $\mu(G):=m(G)-m(G / N)$.

Given a positive integer $n$ and a group $H$, we denote by $H$ wr $\operatorname{Sym}(n)$ the wreath product of $H$ with the symmetric group $\operatorname{Sym}(n)$ of degree $n$. We denote the elements of $H w \operatorname{wym}(n)$ with ordered pairs $f \sigma$, where $f \in H^{n}$ and $\sigma \in \operatorname{Sym}(n)$.

Given two positive integers $x$ and $n$ with $x, n \geq 2$, we say that the prime $r$ is a primitive prime divisor of $x^{n}-1$ if $r$ divides $x^{n}-1$ and $r$ is relatively prime to $x^{i}-1$, for each $i \in\{1, \ldots, n-1\}$. From a celebrated theorem of Zsigmondy [17, either $x^{n}-1$ has a primitive prime divisor, or $n=6$ and $x=2$, or $n=2$ and $x+1$ is a power of 2 . In the latter case, when $x$ is a prime power, we deduce that $x$ must be a (Mersenne) prime. We actually need the following refinement. The prime $r$ is said to be a large primitive prime divisor of $x^{n}-1$ if $r$ is a primitive prime divisor of $x^{n}-1$ and either $r>n+1$ or $r^{2}$ divides $x^{n}-1$. We recall the classical result of Feit [6] on the existence of large primitive prime divisors. (We refer also to [15, for an elementary proof of this result.)

Lemma 2.1. If $x$ and $n$ are integers greater than 1 there there exists a large primitive prime divisor for $x^{n}-1$ except exactly in the following cases:

(1) $n=2$ and $x=2^{s} 3^{t}-1$ for some natural numbers $s \geq 0$ and $t \in\{0,1\}$ with $s \geq 2$ if $t=0$,

(2) $x=2$ and $n \in\{4,6,10,12,18\}$,

(3) $x=3$ and $n \in\{4,6\}$,

(4) $x=5$ and $n=6$.

Our last two definitions are rather technical and (for our application) they only pertain to almost simple groups, but they will prove useful. Given an almost simple group $H$ with socle $S$ and a subgroup $K$ of $H$ with $H=K S$, let

$$
t(H, K)
$$

be the smallest cardinality of a set $X$ of $p p$-elements in $S$ with $H=\langle K, X\rangle$. Then, define

$$
t(H):=\max \{t(H, K) \mid K \leq H \text { with } H=K S\} .
$$

From [9, Theorem 1], $S$ is generated by an involution and by an element of odd prime power order and hence

$$
t(H) \leq 2 .
$$

Given a subgroup $K$ of $H$, we say that a subset $Y$ of $H$ is $K$-generating for $H$ if $H=\langle K, Y\rangle$. A $K$-generating set for $H$ is said to be $K$-independent if no proper subset of $Y$ generates $H$ together with $K$. We denote by

$$
m_{K}(H)
$$

the largest cardinality of a $K$-independent generating set for $H$.

2.2. A (short) walk through fixed point ratios and spreads. Let $H$ be an almost simple group with socle $S$ and let $g, s \in H$. We set

$$
P(g, s):=\frac{\left|\left\{t \in s^{H} \mid\langle g, t\rangle \nsupseteq S\right\}\right|}{\left|s^{H}\right|} .
$$

This definition is strictly related to the definition of spread and uniform spread in almost simple groups and we refer the reader to [3, 8] for further details.

For any action of $H$ on a set $\Omega$ and for any $g \in H$, consider the set $\operatorname{Fix}_{\Omega}(g):=\left\{\omega \in \Omega \mid \omega^{g}=\omega\right\}$ of fixed points of $g$ on $\Omega$ and the fixed point ratio

$$
\mu(g, \Omega):=\frac{\left|\operatorname{Fix}_{\Omega}(g)\right|}{|\Omega|} .
$$

From [8, Section 2], if $M \backslash H$ denotes the set of right cosets of the subgroup $M$ of $H$, then

$$
\mu(g, M \backslash H)=\frac{\left|g^{H} \cap M\right|}{\left|g^{H}\right|} .
$$


Let now $\mathcal{M}(H, g)$ be the collection of all maximal subgroups of $H$ containing $g$ and assume that $H$ is almost simple with socle $S$. Then, from (2.2), we deduce

$$
P(g, s) \leq \sum_{M \in \mathcal{M}(H, g)} \frac{\left|\left\{t \in s^{H} \mid\langle g, t\rangle \leq M\right\}\right|}{\left|s^{H}\right|}=\sum_{M \in \mathcal{M}(H, s)} \frac{\left|\left\{h \in g^{H} \mid\langle h, s\rangle \leq M\right\}\right|}{\left|g^{H}\right|} \leq \sum_{M \in \mathcal{M}(H, s)} \mu(g, M \backslash H) .
$$

Eq. (2.3) also appears in [3, (2.4)]. We summarize in the following lemma the main application of fixed point ratios in our context.

Lemma 2.2. Let $H$ be an almost simple group with socle $S$. Suppose $H \neq S$. If, for every $g \in H \backslash S$, there exists a pp-element $s_{g} \in S$ with $P\left(g, s_{g}\right)<1$, then $t(H)=1$. In particular, if $\sum_{M \in \mathcal{M}(H, s)} \mu(g, M \backslash H)<1$ for every $g \in H \backslash S$, then $t(H)=1$.

Proof. Let $K$ be a subgroup of $H$ with $H=K S$. For every $g \in K \backslash S$, let $s_{g}$ be a $p p$-element belonging to $S$ with $P\left(g, s_{g}\right)<1$. Then by definition of $P\left(g, s_{g}\right)$, there exists $t \in s_{g}^{H}$ with $\langle g, t\rangle \geq S$. Thus $H=\langle K, t\rangle$ and hence $t(H, K)=1$. Since this holds regardless of $K$, we have $t(H)=1$. The rest of the proof follows from (2.3).

\subsection{Basic results.}

Lemma 2.3. Let $G$ be a group. Then $m(G)=m_{p p}(G)$.

Proof. As we have observed above, $m(G) \geq m_{p p}(G)$ and hence we only need to show that $m(G) \leq m_{p p}(G)$.

Let $X:=\left\{x_{1}, \ldots, x_{m(G)}\right\}$ be an independent generating set for $G$ of cardinality $m(G)$. For each $i \in\{1, \ldots, m(G)\}$, we may write $x_{i}=y_{1, i} \cdots y_{k_{i}, i}$, where $y_{1, i}, \ldots, y_{k_{i}, i}$ are pair-wise commuting $p p$-elements of $G$ with

$$
\left\langle x_{i}\right\rangle=\left\langle y_{1, i}, \ldots, y_{k_{i}, i}\right\rangle .
$$

Clearly,

$$
\left\{y_{j, i} \mid 1 \leq j \leq k_{i}, 1 \leq i \leq m(G)\right\}
$$

is a generating set for $G$ consisting of $p p$-elements and hence it contains a $p p$-base $Y$.

We claim that, for each $i \in\{1, \ldots, m(G)\}$, there exists $j \in\left\{1, \ldots, k_{i}\right\}$ with $y_{j, i} \in Y$. Indeed, if for some some $\bar{i}, Y$ contains no $y_{j, \bar{i}}$, then

$$
G=\langle Y\rangle \leq\left\langle y_{j, i} \mid i \in\{1, \ldots, m(G)\} \backslash\{\bar{i}\}, j \in\left\{1, \ldots, k_{i}\right\}\right\rangle \leq\left\langle X \backslash\left\{x_{\bar{i}}\right\}\right\rangle,
$$

where in the last inequality we have used (2.4). However, this contradicts the fact that $X$ is independent and hence the claim is proved.

The previous paragraph yields $|Y| \geq m(G)$ and hence the lemma follows because $m_{p p}(G) \geq|Y|$.

We now recall [10, Theorem $6.1(1)$ ].

Lemma 2.4. If $G$ is a $\mathcal{B}_{p p}$-group, then every quotient of $G$ is a $\mathcal{B}_{p p}$-group.

\section{Proofs of Theorem 1.1 and Corollary 1.2}

\subsection{Technical lemmas.}

Lemma 3.1. Let $q$ be a prime power with $q \geq 4$ and let $H$ be an almost simple group with socle $S:=\mathrm{PSL}_{2}(q)$ and with $H \neq S$. Then $t(H)=1$.

Proof. It suffices to prove that, for every subgroup $K$ of $H$ with $H=K S$, there exists a $p p$-element $x_{K} \in S$ with $H=\left\langle K, x_{K}\right\rangle$. Write $q:=p^{f}$, where $p$ is a prime number and $f$ is a positive integer.

Let $K \leq H$ with $H=K S$ and let $\theta \in K \backslash S$. Assume that $p^{2 f}-1$ admits no large primitive prime divisor. From Lemma 2.1 we deduce that either

$$
S \in\left\{\mathrm{PSL}_{2}(4)=\mathrm{PSL}_{2}(5), \mathrm{PSL}_{2}(8), \mathrm{PSL}_{2}(32), \mathrm{PSL}_{2}(64), \mathrm{PSL}_{2}(512), \mathrm{PSL}_{2}(9), \mathrm{PSL}_{2}(27), \mathrm{PSL}_{2}(125)\right\}
$$

or $f=1$ and $q=p=2^{s} 3^{t}-1$ for some natural numbers $s \geq 0$ and $t \in\{0,1\}$ with $s \geq 2$ if $t=0$. In the first eight exceptional cases, the result can be established with a direct inspection using, for instance, the assistance of the computer algebra system magma [1. We now consider the case $q=p=2^{s} 3^{t}-1$. Actually, we deal with the more general case that $q=p$ is a prime number. As $H \neq S$, we have $H=\operatorname{PGL}_{2}(q)$. Clearly, a Sylow $p$-subgroup of $S$ is cyclic; let $x \in S$ be an element generating a Sylow $p$-subgroup of $S$. Observe that we may choose $x$ so that $\theta$ does not normalize $\langle x\rangle$. Using the list of the maximal subgroups of $S$ (see for instance [2, Tables 8.1, 8.2]), we see that $S=\left\langle x, x^{\theta}\right\rangle$. Thus $H=\langle K, x\rangle$ and $t(H, K)=1$.

Assume now that $p^{2 f}-1$ admits a large primitive prime divisor $r$. Observe that, from the previous paragraph, we may suppose that $f>1$. In particular, either $r>2 f+1 \geq 5$ or $r^{2}$ divides $q+1$. Clearly, a Sylow $r$-subgroup of $S$ is cyclic; let $x \in S$ be an element generating a Sylow $r$-subgroup of $S$. Observe that we may choose $x$ so that $\theta$ does not normalize $\langle x\rangle$ (this can be easily established by considering the structure of the subgroup lattice of $S$, see [2, Table 8.1]). Using the list of the maximal subgroups of $S$ (see for instance [2, Tables 8.1, 8.2]), we see that either 
- $S=\left\langle x, x^{\theta}\right\rangle$, or

- $r=5$ and $\left\langle x, x^{\theta}\right\rangle \cong \operatorname{Alt}(5)$, or

- $r=3$ and $\left\langle x, x^{\theta}\right\rangle$ is isomorphic to either Alt(4) or Alt(5).

In the first case, $H=\langle K, x\rangle$ and hence $t(H, K)=1$. In the last two cases, $r$ is the cardinality of a Sylow $r$-subgroup of $S$, because 5 is the cardinality of a Sylow 5-subgroup of Alt(5) and 3 is the cardinality of a Sylow 3-subgroup of Sym(4). However, this contradicts the fact that $r$ is a large primitive prime divisor of $p^{2 f}-1$.

Lemma 3.2. Let $q$ be a prime power and let $H$ be an almost simple group with socle $S:=\mathrm{PSU}_{3}(q)$ and with $H \neq S$. Then $t(H)=1$.

Proof. As $\mathrm{PSU}_{3}(2)$ is solvable, we have $q>2$. Here the argument is similar to the proof of Lemma 3.1 we use primitive prime divisors and the structure of the subgroup lattice of $S$, see [2, Tables 8.5, 8.6]. Write $q:=p^{f}$, where $p$ is a prime number and $f$ is a positive integer.

Let $K \leq H$ with $H=K S$ and let $\theta \in K \backslash S$. Assume $p^{6 f}-1$ admits a large primitive prime divisor of $r$. Clearly, a Sylow $r$-subgroup of $S$ is cyclic; let $x \in S$ be an element generating a Sylow $r$-subgroup of $S$. Observe that we may choose $x$ so that $\theta$ does not normalize $\langle x\rangle$. Using the list of the maximal subgroups of $S$ (see [2, Tables 8.5, 8.6]), we see that $S=\left\langle x, x^{\theta}\right\rangle$ (here we are using the fact that $r$ is a large Zsigmondy prime and hence $\left\langle x, x^{\theta}\right\rangle$ cannot be contained in a maximal subgroup in the Aschbacher class $\mathcal{S}$ by [2, Table 8.6]). Thus $H=\langle K, x\rangle$ and $t(H, K)=1$.

It remains to consider the case that $p^{6 f}-1$ does not admit a large primitive prime divisor. Lemma 2.1 yields $(f, p) \in$ $\{(1,5),(1,3),(2,2),(3,2)\}$. Here the proof follows with the invaluable help of the computer algebra system magma 1 .

Lemma 3.3. Let $q$ be a prime power and let $H$ be an almost simple group with socle $S:=\operatorname{PSL}_{3}(q)$ and with $S<H \not \leq$ $\mathrm{P \Gamma L}_{3}(q)$. Then $t(H)=1$.

Proof. As $\mathrm{PSL}_{2}(7) \cong \mathrm{PSL}_{3}(2)$, from Lemma 3.1 we may suppose that $q>2$. Here the argument is similar to the proof of Lemma 3.1 we use primitive prime divisors and the structure of the subgroup lattice of $S$, see [2, Tables 8.3, 8.4]. Write $q:=p^{f}$, where $p$ is a prime number and $f$ is a positive integer. As $q>2$, we have $(p, f) \neq(2,1)$.

Let $K \leq H$ with $H=K S$ and let $\theta \in K \backslash S$. From Lemma 2.1, $p^{3 f}-1$ has a large primitive prime divisors, except when $(p, f) \in\{(2,2),(2,4),(2,6),(3,2),(5,2)\}$. For these exceptional cases, we have checked the veracity of this lemma with a computer computation. In particular, for the rest of the argument, we let $r$ be a large primitive prime divisor of $p^{3 f}-1$.

A Sylow $r$-subgroup of $S$ is cyclic; let $x \in S$ be an element generating a Sylow $r$-subgroup of $S$. Let $M \in \mathcal{M}(H, x)$. Here we use the information in [2, Tables 8.3, 8.4]. From the list of the maximal subgroups of $H$ and recalling that $S<H \not \mathrm{P} \mathrm{LL}_{3}(q)$ and $r$ is a large primitive prime divisor, we deduce that either $M=\mathbf{N}_{H}(\langle x\rangle)$, or $f$ is even, $q=q_{0}^{2}$ and $M \cap S \cong \mathrm{SU}_{3}\left(q_{0}\right)$ (here we are using the fact that $r$ is a large Zsigmondy prime and hence $\left\langle x, x^{\theta}\right\rangle$ cannot be contained in a maximal subgroup in the Aschbacher class $\mathcal{S}$ by [2, Table 8.4]). In particular, when $f$ is odd, we have $\mathcal{M}(H, x)=\left\{\mathbf{N}_{H}(\langle x\rangle)\right\}$. Therefore, we deduce

$$
\sum_{M \in \mathcal{M}(H, x)} \mu(\theta, M \backslash H)=\mu\left(\theta, \mathbf{N}_{H}(\langle x\rangle) \backslash H\right)<1,
$$

and hence $t(H, K)=1$, from Lemma 2.2 .

Suppose now that $f$ is even and let $\bar{M} \in \mathcal{M}(H, x) \backslash\left\{\mathbf{N}_{H}(\langle x\rangle)\right\}$. Then $\bar{M} \cap S \cong \mathrm{SU}_{3}\left(q_{0}\right)$, where $q=q_{0}^{2}=p^{f / 2}$. Observe that from the "c" column in [2, Table 8.42], we deduce that the maximal subgroups of $H$ with $\bar{M} \cap S$ isomorphic to $\mathrm{SU}_{3}\left(q_{0}\right)$ form $\operatorname{gcd}\left(q_{0}-1,3\right) S$-conjugacy class. Let $\Omega_{1}:=\left\{\left\langle x^{g}\right\rangle \mid g \in H\right\}$. Using the information in [2, Table 8.3], we deduce

$$
\left|\Omega_{1}\right|=\frac{q^{3}\left(q^{3}-1\right)\left(q^{2}-1\right)}{\left(q^{2}+q+1\right) 3}=\frac{q^{3}\left(q^{2}-1\right)(q-1)}{3} .
$$

Let $\Omega_{2}:=\left\{\bar{M}^{g} \mid g \in H\right\}$. Using the information in [2, Table 8.3], we deduce

$$
\left|\Omega_{2}\right|=\frac{q^{3}\left(q^{3}-1\right)\left(q^{2}-1\right)}{\left(q_{0}^{3}+1\right) q_{0}^{3}\left(q_{0}^{2}-1\right)}=q_{0}^{3}\left(q_{0}^{3}-1\right)\left(q_{0}^{2}+1\right) .
$$

How, consider the bipartite graph having vertex set $\Omega_{1} \cup \Omega_{2}$ and having edge set consisting of the pairs $\{A, B\}$ with $A \in \Omega_{1}, B \in \Omega_{2}$ and $A \leq B$. Fix $B \in \Omega_{2}$. Using the structure of the unitary group $B$, we see that the number of $A \in \Omega_{1}$ with $A \leq B$ is

$$
\frac{\left(q_{0}^{3}+1\right) q_{0}^{3}\left(q_{0}^{2}-1\right)}{\left(q_{0}^{2}-q_{0}+1\right) 3}=\frac{q_{0}^{3}\left(q_{0}^{2}-1\right)\left(q_{0}+1\right)}{3} .
$$

In particular, the number of edges of the bipartite graph is

$$
\left|\Omega_{2}\right| \frac{q_{0}^{3}\left(q_{0}^{2}-1\right)\left(q_{0}+1\right)}{3}=\frac{q^{3}\left(q^{2}-1\right)\left(q_{0}^{3}-1\right)\left(q_{0}+1\right)}{3} .
$$


This shows that the number of elements in $\Omega_{2}$ containing the element $\bar{M} \in \Omega_{1}$ is

$$
\frac{\frac{q^{3}\left(q^{2}-1\right)\left(q_{0}^{3}-1\right)\left(q_{0}+1\right)}{3}}{\left|\Omega_{1}\right|}=q_{0}^{2}+q_{0}+1 .
$$

Thus

$$
|\mathcal{M}(H, x)|=\left|\left\{\mathbf{N}_{H}(\langle x\rangle)\right\} \cup\left\{M \in \Omega_{2} \mid x \in M\right\}\right|=q_{0}^{2}+q_{0}+2 .
$$

From [5, Lemma 2.10 (ii)], we have $\mu(\theta, M \backslash H) \leq \operatorname{gcd}(3, q-1) /\left(q_{0}(q+1)\right)$ for every $M \in \mathcal{M}(\theta, M \backslash H)$ with $M \cap S \cong$ $\mathrm{SU}_{3}\left(q_{0}\right)$. Moreover, from [12, Theorem 1], we have $\mu\left(\theta, \mathbf{N}_{H}(\langle x\rangle) \backslash H\right) \leq 4 /(3 q)$. Therefore

$$
\sum_{M \in \mathcal{M}(H, x)} \mu(\theta, M \backslash H) \leq \operatorname{gcd}(3, q-1) \frac{q_{0}^{2}+q_{0}+1}{q_{0}(q+1)}+\frac{4}{3 q}<1,
$$

whenever $q \notin\{4,16\}$. Since we have excluded the case $q=4$ above, it remains to deal with $q=16$. This case, yet again, has been dealt with a computer computation. Now Lemma 2.2 shows that $t(H)=1$.

Lemma 3.4. Let e be a positive integer, let $q=3^{2 e+1}$ and let $H$ be an almost simple group with socle $S:={ }^{2} G_{2}(q)$ and with $H \neq S$. Then $t(H)=1$.

Proof. Let $K \leq H$ with $H=K S$ and let $\theta \in K \backslash S$. Let $r$ be a primitive prime divisor of $q^{6}-1$. From the structure of the Ree groups ${ }^{2} G_{2}(q)$, we deduce that the Sylow $r$-subgroups of $S$ are cyclic. Let $x \in S$ be an element generating a Sylow $r$-subgroup of $S$. Using the list of the maximal subgroups of $S$ [2, Tables 8.43], we deduce that $|\mathcal{M}(H, x)|=1$. Indeed, $\mathcal{M}(H, x)=\left\{\mathbf{N}_{H}(\langle x\rangle)\right\}$. From (2.3), we have $P(\theta, x) \leq \mu\left(\theta, \mathbf{N}_{H}(\langle x\rangle) \backslash H\right)<1$. Now Lemma 2.2 shows that $t(H)=1$.

Lemma 3.5. Let e be a positive integer, let $q=2^{2 e+1}$ and let $H$ be an almost simple group with socle $S:={ }^{2} B_{2}(q)$ and with $H \neq S$. Then $t(H)=1$.

Proof. Let $K \leq H$ with $H=K S$ and let $\theta \in K \backslash S$. Let $r$ be a primitive prime divisor of $q^{4}-1$. From the structure of the Suzuki groups ${ }^{2} B_{2}(q)$, we deduce that the Sylow $r$-subgroups of $S$ are cyclic. Let $x \in S$ be an element generating a Sylow $r$-subgroup of $S$. Using the list of the maximal subgroups of $S$ [2, Tables 8.16], we deduce that $|\mathcal{M}(H, x)|=1$ and $\mathcal{M}(H, x)=\left\{\mathbf{N}_{H}(\langle x\rangle)\right\}$. Now, the proof follows as in the proof of Lemma 3.4 .

Lemma 3.6. Let e be a positive integer with $e \geq 1$, let $q=3^{e}$ and let $H$ be an almost simple group with socle $S:=G_{2}(q)$ and with $H$ containing an outer automorphism which is not a field automorphism. Then $t(H)=1$.

Proof. Recall that $|\operatorname{Aut}(S): S|=2 e$. When $e=1$, we have checked the veracity of this lemma with the computer algebra system magma [1]. Therefore for the rest of the argument we suppose $e \geq 2$.

Let $K \leq H$ with $H=K S$ and let $\theta \in K \backslash S$. Let $r$ be a primitive prime divisor of $q^{6}-1$. From the structure of the Lie group $G_{2}(q)$, we deduce that the Sylow $r$-subgroups of $S$ are cyclic. Let $x \in S$ be an element generating a Sylow $r$-subgroup of $S$. Let $M \in \mathcal{M}(H, x)$. Here we use the information in [2, Table 8.42]. From the list of the maximal subgroups of $H$ and recalling that $H$ does contain an outer automorphism which is not a field automorphism, we deduce that either $M=\mathbf{N}_{H}(\langle x\rangle)$, or $e$ is odd and $M \cap S \cong{ }^{2} G_{2}(q)$ (here we are assuming $e \geq 2$ ). In particular, when $e$ is even, we have $\mathcal{M}(H, x)=\left\{\mathbf{N}_{H}(\langle x\rangle)\right\}$. Therefore, we deduce

$$
\sum_{M \in \mathcal{M}(H, x)} \mu(\theta, M \backslash H)=\mu\left(\theta, \mathbf{N}_{H}(\langle x\rangle) \backslash H\right)<1,
$$

and hence $t(H, K)=1$, from Lemma 2.2 .

Suppose now that $e$ is odd and let $\bar{M} \in \mathcal{M}(H, x) \backslash\left\{\mathbf{N}_{H}(\langle x\rangle)\right\}$. Then $\bar{M} \cap S \cong{ }^{2} G_{2}(q)$. Observe that from the "c" column in [2, Table 8.42], we deduce that the maximal subgroups of $H$ with $\bar{M} \cap S$ isomorphic to ${ }^{2} G_{2}(q)$ form a unique conjugacy class. Observe that

$$
q^{6}-1=\left(q^{3}-1\right)(q+1)(q+\sqrt{3 q}+1)(q-\sqrt{3 q}+1) .
$$

In particular, the primitive prime divisor $r$ of $q^{6}-1$ can be chosen so that $r$ divides $q+\sqrt{3 q}+1$. Let $\Omega_{1}:=\left\{\left\langle x^{g}\right\rangle \mid g \in H\right\}$. Using the information in [2, Table 8.42], we deduce

$$
\left|\Omega_{1}\right|=\frac{q^{6}\left(q^{6}-1\right)\left(q^{2}-1\right)}{\left(q^{2}-q+1\right) 6}=\frac{q^{6}\left(q^{3}-1\right)\left(q^{2}-1\right)(q+1)}{6} .
$$

Let $\Omega_{2}:=\left\{\bar{M}^{g} \mid g \in H\right\}$. Using the information in [2, Table 8.42], we deduce

$$
\left|\Omega_{2}\right|=\frac{q^{6}\left(q^{6}-1\right)\left(q^{2}-1\right)}{\left(q^{3}+1\right) q^{3}(q-1)}=q^{3}\left(q^{3}-1\right)(q+1) .
$$


Now, consider the bipartite graph having vertex set $\Omega_{1} \cup \Omega_{2}$ and having edge set consisting of the pairs $\{A, B\}$ with $A \in \Omega_{1}, B \in \Omega_{2}$ and $A \leq B$. Fix $B \in \Omega_{2}$. Using the structure of the Ree group $B$, we see that the number of $A \in \Omega_{1}$ with $A \leq B$ is

$$
\frac{\left(q^{3}+1\right) q^{3}(q-1)}{(q+\sqrt{3 q}+1) 6}=\frac{(q-\sqrt{3 q}+1) q^{3}\left(q^{2}-1\right)}{6} .
$$

In particular, the number of edges of the bipartite graph is

$$
\left|\Omega_{2}\right| \frac{(q-\sqrt{3 q}+1) q^{3}\left(q^{2}-1\right)}{6}=\frac{q^{6}\left(q^{3}-1\right)\left(q^{2}-1\right)(q-\sqrt{3 q}+1)(q+1)}{6} .
$$

This shows that the number of elements in $\Omega_{2}$ containing the element $\bar{M} \in \Omega_{1}$ is

$$
\frac{q^{6}\left(q^{3}-1\right)\left(q^{2}-1\right)(q-\sqrt{3 q}+1)(q+1)}{6}=q-\sqrt{3 q}+1 \text {. }
$$

Thus

$$
|\mathcal{M}(H, x)|=\left|\left\{\mathbf{N}_{H}(\langle x\rangle)\right\} \cup\left\{M \in \Omega_{2} \mid x \in M\right\}\right|=q-\sqrt{3 q}+2 .
$$

From [11, Theorem 1], we have $\mu(\theta, M \backslash H)<1 /\left(q^{2}-q+1\right)$ for every $M \in \mathcal{M}(\theta, M \backslash H)$. Therefore

$$
\sum_{M \in \mathcal{M}(H, x)} \mu(\theta, M \backslash H) \leq \frac{q-\sqrt{3 q}+2}{q^{2}-q+1}<1 .
$$

Now Lemma 2.2 shows that $t(H)=1$.

Lemma 3.7. Let e be a positive integer with $e \geq 2$, let $q=2^{e}$ and let $H$ be an almost simple group with socle $S:=\operatorname{Sp}_{4}(q)$ and with $H$ containing an outer automorphism which is not a field automorphism. Then $t(H)=1$.

Proof. Recall that $|\operatorname{Aut}(S): S|=2 e$. Let $K \leq H$ with $H=K S$ and let $\theta \in K \backslash S$. Let $r$ be a primitive prime divisor of $q^{4}-1$. From the structure of the classical group $\operatorname{Sp}_{4}(q)$, we deduce that the Sylow $r$-subgroups of $S$ are cyclic. Let $x \in S$ be an element generating a Sylow $r$-subgroup of $S$.

Let $M \in \mathcal{M}(H, x)$. Here we use the information in [2, Table 8.14]. From the list of the maximal subgroups of $H$ and recalling that $H$ does contain an outer automorphism which is not a field automorphism, we deduce that either $M=\mathbf{N}_{H}(\langle x\rangle)$, or $e$ is odd and $M \cap S \cong{ }^{2} B_{2}(q)$. In particular, when $e$ is even, we have $\mathcal{M}(H, x)=\left\{\mathbf{N}_{H}(\langle x\rangle)\right\}$. Therefore, we deduce

$$
\sum_{M \in \mathcal{M}(H, x)} \mu(\theta, M \backslash H)=\mu\left(\theta, \mathbf{N}_{H}(\langle x\rangle) \backslash H\right)<1,
$$

and hence $t(H, K)=1$, from Lemma 2.2 .

Suppose now that $e$ is odd and let $\bar{M} \in \mathcal{M}(H, x) \backslash\left\{\mathbf{N}_{H}(\langle x\rangle)\right\}$. Then $\bar{M} \cap S \cong{ }^{2} B_{2}(q)$. Observe that from the "c" column in [2, Table 8.14], we deduce that the maximal subgroups of $H$ with $\bar{M} \cap S$ isomorphic to ${ }^{2} B_{2}(q)$ form a unique conjugacy class. Observe that

$$
q^{4}-1=\left(q^{2}-1\right)(q+\sqrt{2 q}+1)(q-\sqrt{2 q}+1) .
$$

In particular, the primitive prime divisor $r$ of $q^{4}-1$ can be chosen so that $r$ divides $q+\sqrt{2 q}+1$. Let $\Omega_{1}:=\left\{\left\langle x^{g}\right\rangle \mid g \in H\right\}$. Using the information in [2, Table 8.14], we deduce

$$
\left|\Omega_{1}\right|=\frac{q^{4}\left(q^{4}-1\right)\left(q^{2}-1\right)}{\left(q^{2}+1\right) 4}=\frac{q^{4}\left(q^{2}-1\right)^{2}}{4} .
$$

Let $\Omega_{2}:=\left\{\bar{M}^{g} \mid g \in H\right\}$. Using the information in [2, Table 8.14], we deduce

$$
\left|\Omega_{2}\right|=\frac{q^{4}\left(q^{4}-1\right)\left(q^{2}-1\right)}{\left(q^{2}+1\right) q^{2}(q-1)}=q^{2}\left(q^{2}-1\right)(q+1) .
$$

How, consider the bipartite graph having vertex set $\Omega_{1} \cup \Omega_{2}$ and having edge set consisting of the pairs $\{A, B\}$ with $A \in \Omega_{1}, B \in \Omega_{2}$ and $A \leq B$. Fix $B \in \Omega_{2}$. Using the structure of the Suzuki group $B$, we see that the number of $A \in \Omega_{1}$ with $A \leq B$ is

$$
\frac{\left(q^{2}+1\right) q^{2}(q-1)}{(q+\sqrt{2 q}+1) 4}=\frac{(q-\sqrt{2 q}+1) q^{2}(q-1)}{4} .
$$

In particular, the number of edges of the bipartite graph is

$$
\left|\Omega_{2}\right| \frac{(q-\sqrt{2 q}+1) q^{2}(q-1)}{4}=\frac{q^{4}\left(q^{2}-1\right)^{2}(q-\sqrt{2 q}+1)}{4} .
$$

This shows that the number of elements in $\Omega_{2}$ containing the element $\bar{M} \in \Omega_{1}$ is

$$
\frac{q^{4}\left(q^{2}-1\right)^{2}(q-\sqrt{2 q}+1)}{4}=q-\sqrt{2 q}+1 \text {. }
$$


Thus

$$
|\mathcal{M}(H, x)|=\left|\left\{\mathbf{N}_{H}(\langle x\rangle)\right\} \cup\left\{M \in \Omega_{2} \mid x \in M\right\}\right|=q-\sqrt{2 q}+2 .
$$

Now, 4, Theorem 1] yields $\mu(\theta, M \backslash H) \leq\left|\theta^{H}\right|^{-\frac{1}{4}}=\left|H: \mathbf{C}_{H}(\theta)\right|^{-\frac{1}{4}}$ for every $M \in \mathcal{M}(H, x)$. As $\theta$ is an outer automorphism which is not a field automorphism and as $e$ is odd, replacing $\theta$ with a suitable power, we may suppose that $\theta$ is an involution and that $\theta$ is a graph-field automorphism. From [7, we deduce that $\mathbf{C}_{S}(\theta) \cong{ }^{2} B_{2}(q)$ and hence

$$
\left|\theta^{H}\right|=\frac{q^{4}\left(q^{4}-1\right)\left(q^{2}-1\right)}{\left(q^{2}+1\right) q^{2}(q-1)}=q^{2}\left(q^{2}+1\right)(q+1) .
$$

Therefore

$$
\sum_{M \in \mathcal{M}(M, x)} \mu(\theta, M \backslash H) \leq \frac{q-\sqrt{2 q}+2}{\left(q^{2}\left(q^{2}+1\right)(q+1)\right)^{1 / 4}}<1,
$$

where the last inequality follows with a computation. Now Lemma 2.2 shows that $t(H)=1$.

Lemma 3.8. Let $H$ be an almost simple group with socle $S$. Then there exists a subgroup $K$ of $H$ with $H=K S$ and with $m_{K}(H)>t(H)$.

Proof. Suppose first $H=S$. Choose $K:=1$. Then $m_{K}(H)=m(H) \geq 3$, because we can generate $H=S$ with conjugated involutions. Therefore, the proof follows from (2.1). Thus, for the rest of the argument, we suppose $H \neq S$. Now, we use the Classification of Finite Simple Groups and we divide our proof depending on the type of $S$.

Alternating groups: Suppose $S$ is an alternating group $\operatorname{Alt}(n)$ of degree $n \geq 5$. Assume first $n \neq 6$, or $n=6$ and $H=\operatorname{Sym}(6)$. Then $H=\operatorname{Sym}(n)$. Choose $K:=\langle(1,2)\rangle$ and let

$$
\Lambda:=\{(1,2,3),(1,2)(3,4),(1,2)(3,5), \ldots,(1,2)(3, n)\} .
$$

It is readily seen that $\Lambda$ is a $K$-independent generating set for $H$. Therefore, $m_{K}(H) \geq|\Lambda|=n-2 \geq 3$ and the proof follows again from (2.1).

As Alt(6) $\cong \mathrm{PSL}_{2}(9)$, we postpone the proof of the case $n=6$ and $H \neq \operatorname{Sym}(6)$, when we deal with groups of Lie type. Sporadic Groups: Suppose $S$ is a sporadic simple group. As $H \neq S$, we deduce $H=$ Aut $S$ and $S$ is one of the following groups

$$
F i_{22}, F i_{24}, H N, J_{3}, M_{22}, O^{\prime} N, H S, J_{2}, M c L, H e, M_{12}, S u z .
$$

If $S \in\left\{F i_{22}, F i_{24}, H N, J_{3}, M_{22}, O^{\prime} N\right\}$, then it follows from [3, Table 9] that $t(H)=1$. However, if we choose $\alpha$ an involution from $H \backslash S$ and we set $K:=\langle\alpha\rangle$, then $m_{K}(H) \geq 2$, because we can generated $H$ with $\alpha$ and a suitable number (at least 2) of involutions from $S$.

If $S \in\left\{H S, J_{2}, M c L, H e, M_{12}, S u z\right\}$, we have verified that $m_{K}(H) \geq 3$ using magma: in all cases there exists $\alpha \in H \backslash S$ with $|\alpha|=2$ and three conjugated involutions in $S$ such that $\left\{\alpha, t_{1}, t_{2}, t_{3}\right\}$ is a $\langle\alpha\rangle$-independent generating set for $H$.

Groups of LiE TYPE: Here we use the information and the notation in [7, Section 2.4]. The simple group of Lie type $S$ is generated by root elements $x_{ \pm \hat{\alpha}}(t)$, where $\alpha \in \Pi, \Pi$ is a fundamental system for the root system $\Sigma$ of $S$, and $t$ lies in a suitable finite field $\mathbb{F}$. As $x_{\hat{\alpha}}(t)$ is unipotent, $x_{\hat{\alpha}}(t)$ has prime order and hence it is a $p p$-element.

The action of the automorphism group of $S$ on the root elements $x_{ \pm \hat{\alpha}}(t)$ is described in [7, Section 2.5] and again we use the information and the notation therein. The outer automorphisms of $S$ are divided in inner-diagonal, field and graph automorphisms. These can be chosen so that inner-diagonal and field automorphisms normalize each root subgroup $\left\langle x_{\hat{\alpha}}(t) \mid t \in \mathbb{F}\right\rangle$; whereas, graph automorphisms permute the root subgroups according to the action of the graph automorphism on the nodes of the Dynkin diagram. In particular, we may choose a supplement $K$ of $S$ in $H$ so that the elements in $K$ consist of inner-diagonal, field and graph automorphisms, with respect to the choice of the root system $\Sigma$. Now, let $\tilde{\Pi} \subseteq \Pi$ be a set of representatives of the orbits for the action of $K$ on $\Pi$. Then

$$
H=\left\langle K, x_{\hat{\alpha}}(t) \mid \alpha \in \pm \tilde{\Pi}, t \in \mathbb{F}\right\rangle
$$

and hence from the set $\left\{x_{\hat{\alpha}}(t) \mid \alpha \in \pm \tilde{\Pi}, t \in \mathbb{F}\right\}$ we may extract a $K$-independent generating set $Y$ for $H$ consisting of $p p$-elements. For each $\beta \in \pm \Pi$, define $S_{\beta}:=\left\langle x_{\hat{\alpha}}(t) \mid \alpha \in \pm \Pi \backslash\{\beta\}, t \in \mathbb{F}\right\rangle$. Observe that $S_{\beta}$ is contained in a proper parabolic subgroup of $S$ normalized by $K$. This implies $|Y| \geq 2|\tilde{\Pi}|$. A direct inspection on the various root systems gives that one of the following holds:

(1) $|\tilde{\Pi}| \geq 2$, or

(2) $S$ is a simple group of Lie type of Lie rank 1, that is, $S \in\left\{A_{1}(q)=\mathrm{PSL}_{2}(q),{ }^{2} A_{2}(q)=\operatorname{PSU}_{3}(q),{ }^{2} B_{2}(q),{ }^{2} G_{2}(q)\right\}$, or

(3) $S=A_{2}(q)=\mathrm{PSL}_{3}(q)$ and $H \not \leq \mathrm{P}_{3}(q)$,

(4) $S=B_{2}(q)=\operatorname{PSp}_{4}(q), q=2^{e}$ for some $e \geq 1$ and $H$ contains an outer automorphism which is not a field automorphism,

(5) $S=G_{2}(q), q=3^{e}$ for some $e \geq 1$, and $H$ contains an outer automorphism which is not a field automorphism. 
If (1) holds, then the proof follows from (2.1). In the remaining cases, we have shown in Lemmas 3.1, 3.2, 3.3, 3.4, 3.5, 3.6 and 3.7 that $t(H)=1$. Using this slight refinement on the value of $t(H)$ and repeating the argument above for the remaining groups we deduce $m_{K}(H) \geq 2>1=t(H)$.

\subsection{Pulling the threads of the argument.}

Proof of Theorem 1.1. We argue by contradiction and among all non-soluble $\mathcal{B}_{p p}$-groups we choose $G$ having minimal order.

Let $N$ be a minimal normal subgroup of $G$. From Lemma 2.4, $G / N$ is a $\mathcal{B}_{p p}$-group and hence, from our minimal choice of $G$, we deduce that

$$
G / N \text { is solvable. }
$$

Suppose that $G$ has two distinct minimal normal subgroups $N_{1}$ and $N_{2}$. Since $N_{1} \cap N_{2}=1, G$ embeds into the cartesian product $G / N_{1} \times G / N_{2}$. As $G / N_{1}$ and $G / N_{2}$ are both solvable, we deduce that $G$ is solvable, which is a contradiction. Therefore, $G$ has a unique minimal normal subgroup $N$, that is, $G$ is monolithic.

If $N$ is abelian, then $G$ is solvable by (3.1), which is a contradiction. Therefore, $N$ is non-abelian and hence $N \cong S^{n}$, for some non-abelian simple group $S$. Write $N:=S_{1} \times \cdots \times S_{n}$, where $S_{1}, \ldots, S_{n}$ are the simple direct factors of $N$. Let $H$ be the subgroup of Aut $(S)$ induced by the conjugacy action of $\mathbf{N}_{G}\left(S_{1}\right)$ on $S$. Clearly, $H$ is an almost simple group with socle $S$. Moreover, since $G$ is monolithic, $G$ embeds into the wreath product $H$ < $\operatorname{Sym}(n)$ and hence, without loss of generality, we may assume that $G$ is a subgroup of $H \operatorname{wr} \operatorname{Sym}(n)$ with $S^{n} \leq G$ and with

$$
\pi: \mathbf{N}_{G}\left(S_{1}\right) \rightarrow H
$$

projecting onto $H$. In particular, we may write the elements of $G$ as ordered pairs $f \sigma$, with $f \in H^{n}$ and $\sigma \in \operatorname{Sym}(n)$. Let

$$
m_{1}=m(G / N)
$$

Let

$$
Y=\left\{g_{1}, \ldots, g_{m_{1}}\right\}
$$

be a set of $p p$-elements of $G$ with $\left\{g_{1} N, \ldots, g_{m_{1}} N\right\}$ a $p p$-base for $G / N$.

Let

$$
K:=\pi\left(\mathbf{N}_{\langle Y\rangle}\left(S_{1}\right)\right)
$$

As $G=\langle Y\rangle N$, from the modular law we get

$$
\mathbf{N}_{G}\left(S_{1}\right)=\mathbf{N}_{G}\left(S_{1}\right) \cap G=\left(\mathbf{N}_{G}\left(S_{1}\right) \cap\langle Y\rangle\right) N=\mathbf{N}_{\langle Y\rangle}\left(S_{1}\right) N
$$

Thus

$$
H=\pi\left(\mathbf{N}_{G}\left(S_{1}\right)\right)=\pi\left(\mathbf{N}_{\langle Y\rangle}\left(S_{1}\right)\right) \pi(N)=K S .
$$

Let $X$ be a set of $p p$-elements in $S$ with $H=\langle X, K\rangle$ and having cardinality $t(H, K)$. Let

$$
\tilde{X}:=\{(x, \underbrace{1, \ldots, 1}_{n-1 \text { times }}) \in N \mid x \in X\}
$$

and observe that $\tilde{X} \subseteq S^{n}=N \leq G \leq H$ wr $\operatorname{Sym}(n)$.

As $N$ is a minimal normal subgroup of $G, G$ acts transitively by conjugation on the set $\left\{S_{1}, \ldots, S_{n}\right\}$ of simple direct factors of $N$. From this, it follows that $Y \cup \tilde{X}$ is a generating set for $G$. As $Y \cup \tilde{X}$ consists of $p p$-elements and as all $p p$-bases of $G$ have the same cardinality, we get $m_{p p}(G) \leq m_{1}+t(H, K) \leq m_{1}+t(H)$. Thus

$$
m(G) \leq m_{1}+t(H)
$$

by Lemma 2.3 .

Recall the definition of $\mu(G)$ and $\mu(S)$ in Section 2.1] In [14, page 403, inequality (1)] and in [13, Proposition 4], it is proved that $\mu(G) \geq \mu(H)$. Moreover, by [13, Lemma 7], we have $\mu(H) \geq m_{K}(H)$, for every subgroup $K$ of $H$ with $H=K S$. In particular, combining these two results, we deduce $\mu(G) \geq m_{K}(H)$. From (3.2), we get

$$
t(H) \geq m(G)-m_{1}=m(G)-m(G / N)=\mu(G) \geq m_{K}(H)
$$

for every subgroup $K$ of $H$ with $H=K S$. However, this contradicts Lemma 3.8 .

Proof of Corollary 1.2, Let $G$ be a $\mathcal{B}_{p p}$-group with $\Phi(G)=1$. From Theorem 1.1 , $G$ is solvable and hence the proof now follows from [16, Theorem 1.2]. 


\section{Proof of Theorem 1.3}

Let $G$ be a finite group. Take a chief series

$$
1=G_{t} \unlhd \cdots \unlhd G_{0}=G
$$

and consider the non-negative integers $\mu_{i}=m\left(G / G_{i+1}\right)-m\left(G / G_{i}\right)$. Clearly

$$
m(G)=\sum_{0 \leq i \leq t-1} \mu_{i}
$$

Information on the values of $\mu_{i}$ have been obtained in [13, where is it proved in particular:

- if $G_{i} / G_{i+1}$ is abelian, then $\mu_{i}=0$ if $G_{i+1} / G_{i} \leq \Phi\left(G / G_{i+1}\right), \mu_{i}=1$ otherwise;

- if $G_{i} / G_{i+1}$ is non-abelian, then $\mu_{i}=\mu_{i}\left(L_{i}\right)=m\left(L_{i}\right)-m\left(L_{i} / \operatorname{soc} L_{i}\right)$, where $L_{i}=G / C_{G}\left(G_{i} / G_{i+1}\right)$.

In the second case, $L_{i}$ is a monolithic group and $\operatorname{soc} L_{i}=S_{i}^{n_{i}}$ where $n_{i}$ is a positive integer and $S_{i}$ is a finite non-abelian simple group. As we already recalled in the previous section, by [14, page 403, inequality (1)] and [13, Proposition 4], there exists an almost simple group $H_{i}$ such that $\operatorname{soc} H_{i}=S_{i}$ and $\mu_{i}=\mu\left(L_{i}\right) \geq \mu\left(H_{i}\right)$. Moreover, by [13, Lemma 7], we have $\mu\left(H_{i}\right) \geq m_{K_{i}}\left(H_{i}\right)$, for every subgroup $K_{i}$ of $H_{i}$ with $H_{i}=K_{i} S_{i}$. By the results in Section 3, for every choice of $H_{i}$ there exists $K_{i}$ such that $K_{i} S_{i}=H_{i}$ and $m_{K_{i}}\left(H_{i}\right) \geq 2$. So $\mu_{i} \geq 2$ whenever $G_{i} / G_{i+1}$ is non-abelian, and therefore the statement of Theorem 1.3 follows from (4.1).

\section{REFERENCES}

[1] W. Bosma, J. Cannon, C. Playoust, The Magma algebra system. I. The user language, J. Symbolic Comput. 24 (3-4) (1997), $235-265$.

[2] J. H. Bray, D. F. Holt, C. M. Roney-Dougal, The maximal subgroups of the low-dimensional finite classical groups, London Mathematical Society, Lecture Note Series 407, Cambridge University Press, 2013.

[3] T. Breuer, R. M. Guralnick and W. M. Kantor, Probabilistic generation of finite simple groups, II, J. Algebra 320 (2008), 443-494.

[4] T. C. Burness, Fixed point ratios in actions of finite classical groups. I, J. Algebra 309 (2007), 69-79.

[5] T. C. Burness, S. Guest, On the uniform spread of almost simple linear groups, Nagoya Math. J. 209 (2013), 35-109.

[6] W. Feit, On large Zsigmondy primes, Proc. Amer. Math. Soc. 102 (1988), 26-36.

[7] D. Gorenstein, R. Lyons, R. Solomon, The classification of the finite simple groups. number 3. part I. chapter A, 40 (1998), xvi+419.

[8] R. M. Guralnick, W. M. Kantor, Probabilistic generation of finite simple groups, J. Algebra 234 (2000), 743-792.

[9] C. S. H. King, Generation of finite simple groups by an involution and an element of prime order, J. Algebra 478 (2017), $153-173$.

[10] J. Krempa, A. Stocka, On some sets of generators of finite groups, J. Algebra 405 (2014), 122-134.

[11] R. Lawther, M. W. Liebeck, G. M. Seitz, Fixed point ratios in actions of finite exceptional groups of Lie type, Pacific J. Math. 205 (2002), 393-464.

[12] M. W. Liebeck, J. Saxl, Minimal degrees of primitive permutation groups, with an application to monodromy groups of Riemann surfaces, Proc. London Math. Soc. (3) 63 (1991) 266-314.

[13] A. Lucchini, The largest size of a minimal generating set of a finite group, Arch. Math. (Basel) 101 (2013), no. 1, 1-8.

[14] A. Lucchini, Minimal generating sets of maximal size in finite monolithic groups, Arch. Math. (Basel) 101 (2013), no. 5, 401-410.

[15] M. Roitman, On Zsigmondy primes, Proc. Amer. Math. Soc. 125 (1997), 1913-1919.

[16] A. Stocka, Sets of prime power order generators of finite groups, Algebra and Discrete Mathematics 29 (2020), $129-138$.

[17] K. Zsigmondy, Zur Theorie der Potenzreste, Monatsh. Math. Phys. 3 (1892), no. 1, 265-284.

Andrea Lucchini, Dipartimento di Matematica "Tullio Levi-Civita",

University of Padova, Via Trieste 63, 35121 Padova, Italy

Email address: lucchini@math.unipd.it

Pablo Spiga, Dipartimento di Matematica Pura e Applicata,

University of Milano-Bicocca, Via Cozzi 55, 20126 Milano, Italy

Email address: pablo.spiga@unimib.it 